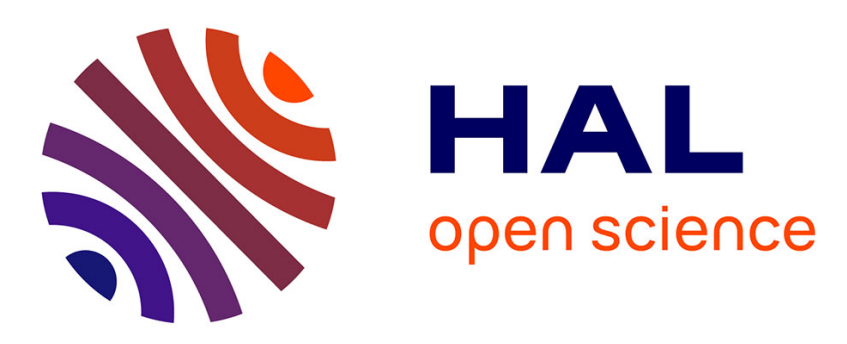

\title{
Statistical model of electron pitch angle diffusion in the outer radiation belt
}

\author{
Anton Artemyev, Oleksiy Agapitov, Vladimir Krasnoselskikh, Hugo \\ Breuillard, Guy Rolland
}

\section{- To cite this version:}

Anton Artemyev, Oleksiy Agapitov, Vladimir Krasnoselskikh, Hugo Breuillard, Guy Rolland. Statistical model of electron pitch angle diffusion in the outer radiation belt. Journal of Geophysical Research Space Physics, 2012, 117, A08219 (10 p.). 10.1029/2012JA017826 . insu-01254458

\section{HAL Id: insu-01254458 \\ https://hal-insu.archives-ouvertes.fr/insu-01254458}

Submitted on 12 Jan 2016

HAL is a multi-disciplinary open access archive for the deposit and dissemination of scientific research documents, whether they are published or not. The documents may come from teaching and research institutions in France or abroad, or from public or private research centers.
L'archive ouverte pluridisciplinaire HAL, est destinée au dépôt et à la diffusion de documents scientifiques de niveau recherche, publiés ou non, émanant des établissements d'enseignement et de recherche français ou étrangers, des laboratoires publics ou privés. 


\title{
Statistical model of electron pitch angle diffusion in the outer radiation belt
}

\author{
A. Artemyev, ${ }^{1,2}$ O. Agapitov, ${ }^{1,3,4}$ V. Krasnoselskikh, ${ }^{1}$ H. Breuillard, ${ }^{1}$ and G. Rolland ${ }^{5}$ \\ Received 12 April 2012; revised 30 June 2012; accepted 3 July 2012; published 21 August 2012.
}

[1] We calculated the bounce averaged electron pitch angle diffusion coefficients $\left\langle D_{\alpha_{e q} \alpha_{e q}}\right\rangle$ using the statistical characteristics of lower band chorus activity collected by the Cluster mission from 2001-2009. Nine years of Cluster observations provide the distributions of the $\theta$ angle between wave vectors and the background magnetic field; and the distributions of the wave total intensity $B_{w}^{2}$ for relatively wide ranges of the magnetic latitude $\lambda$, the magnetic local times, and the $K_{p}$ indices. According to Cluster observations, the probability of observing a larger $B_{w}^{2}$ increases with $\lambda$ and depends upon the magnetic local time and $K_{p}$. We compared the obtained results with the diffusion coefficients $\left\langle D_{\alpha_{e q} \alpha_{e q}}\right\rangle_{\text {const }}$ that were calculated under an assumption of parallel whistler wave propagation with a constant intensity $B_{w}^{2}=10^{4} \mathrm{pT}^{2}$. The last calculations substantially underestimated pitch angle diffusion for the small equatorial pitch angles, $\alpha_{e q}$, but likely overestimates $\left\langle D_{\alpha_{e q} \alpha_{e q}}\right\rangle_{\text {const }}$ for $\alpha_{e q}>60^{\circ}$. An important increase in $\left\langle D_{\alpha_{e q} \alpha_{e q}}\right\rangle$ for $\alpha_{e q}<30^{\circ}$ can be explained by a large mean value for the $\theta$ distribution and by an increase of $B_{w}^{2}$ at $\lambda>15^{\circ}$. We took the probability density distribution of the wave mean amplitude into consideration instead of the averaged value. The obtained distribution of the diffusion coefficients indicated that approximately $20 \%$ of the most intense waves can provide the main portion of pitch angle diffusion for the dawn/day sector. For the dusk/night sector, wave intensity was significantly weaker and the relative importance of intense waves was not clearly pronounced.

Citation: Artemyev, A., O. Agapitov, V. Krasnoselskikh, H. Breuillard, and G. Rolland (2012), Statistical model of electron pitch angle diffusion in the outer radiation belt, J. Geophys. Res., 117, A08219, doi:10.1029/2012JA017826.

\section{Introduction}

[2] Understanding the dynamics of radiation belts is one of the most challenging problems in magnetospheric plasma physics [see Chen et al., 2007; Shprits et al., 2008]. The role of the resonant interaction of electrons and whistler waves for these dynamics was first pointed out by Dungey [1963], and the theoretical approach for the description of this interaction was proposed by Trakhtengerts [1966] and Kennel and Petschek [1966]. In these works the resonant interaction was studied using a quasi-linear diffusion description. The principal elements of such a description are the diffusion coefficients.

[3] The diffusion coefficients were calculated using a quasi-linear approximation [Lyons et al., 1972; Lyons, 1974;

\footnotetext{
${ }^{1}$ LPC2E/CNRS, University of Orleans, Orleans, France.

${ }^{2}$ Space Research Institute, RAS, Moscow, Russia.

${ }^{3}$ National Taras Shevchenko University of Kiev, Kiev, Ukraine.

${ }^{4}$ Le Studium, Institute for Advanced Studies, Orleans, France.

${ }^{5} \mathrm{CNES}$, Toulouse, France.
}

Corresponding author: A. Artemyev, LPC2E/CNRS, University of Orleans, 3A, Avenue de la Recherche Scientifique, FR-45071 Orleans CEDEX, France. (ante0226@gmail.com)

(C)2012. American Geophysical Union. All Rights Reserved. 0148-0227/12/2012JA017826
Glauert and Horne, 2005; Shprits et al., 2006; Summers et al., 2007, and references therein], and are widely used in global modeling, where the Fokker-Plank equation for velocity distributions is considered [Bourdarie et al., 1996; Varotsou et al., 2008; Fok et al., 2008; Albert et al., 2009; Subbotin et al., 2010; Su et al., 2010]. Several analytical (and semi-analytical) schemes for estimating diffusion coefficients have been developed [Summers, 2005; Albert, 2007, 2008; Mourenas and Ripoll, 2012].

[4] To use analytical and numerical schemes, one needs to know the distribution of the wave intensity upon frequency $\hat{B}^{2}(\omega)$, as well as the distribution of the wave propagation direction upon the angle $\theta$ relative to the background magnetic field. The latter distribution is often written as $g(X)$, where $X=\tan \theta$ [Lyons et al., 1972; Lyons, 1974; Glauert and Horne, 2005; Shprits et al., 2006]. According to quasi-linear theory, the diffusion coefficients are proportional to the total wave intensity $B_{w}^{2}=\int \hat{B}^{2}(\omega) d \omega$ (here we defined the mean amplitude as $\left.B_{w}=\sqrt{B_{w}^{2}}\right)$. The $\hat{B}^{2}(\omega), g(X)$ distributions and wave amplitudes can be defined from spacecraft observations [Meredith et al., 2001; Andre et al., 2002; Pokhotelov et al., 2008; Cully et al., 2008; Santolik et al., 2009; Haque et al., 2010; Agapitov et al., 2011, or from numerical calculations of wave propagation in the inner magnetosphere 
[Boskova et al., 1990; Shklyar and Jiřiček, 2000; Jiricek et al., 2001; Bortnik et al., 2008, 2011; Breuillard et al., 2012, and references therein].

[5] Calculation of the diffusion coefficients is often performed using the distribution of the frequency $\hat{B}^{2}(\omega)$ approximated using a Gaussian function [Lyons, 1974; Glauert and Horne, 2005; Summers et al., 2007; Albert, 2007; Shprits and Ni, 2009]. The wave amplitudes are generally considered to be constant for a given $L$-shell and $K_{p}$ index (i.e. $B_{w}$ does not depend on the magnetic latitude $\lambda$ ). The $g(X)$ distribution for the majority of calculations is assumed to be Gaussian with some mean value $X_{m}$ that is close to zero and with a small variance $X_{w}$. One important simplification is an assumption that $X_{m}$ and $X_{w}$ do not depend on $\lambda$, (i.e. $X_{m}, w_{w}$ are constant for a given $L$-shell) [Lyons, 1974; Glauert and Horne, 2005; Summers et al., 2007; Albert, 2007; Shprits and Ni, 2009; Ni et al., 2011].

[6] Recent spacecraft observations [Santolik et al., 2009; Haque et al., 2010; Agapitov et al., 2011; Li et al., 2011] demonstrate that the mean value (as well as the variance) of the $g(X)$ distribution strongly depends on the magnetic latitude $\lambda$ (i.e. one has $g(X) \rightarrow g_{\lambda}(X)$ where $g_{\lambda}(X)$ should be defined for each $\lambda$ ). The effect is very important for the resonant wave-particle interaction [see Shklyar and Matsumoto, 2009, and references therein], and leads to an intensification of the pitch angle diffusion [see, e.g., Shprits and Ni, 2009; Artemyev et al., 2012; Mourenas et al., 2012]. Indeed, electrons with small equatorial pitch angles can reach higher latitudes and their resonant interaction with oblique propagating whistler waves becomes more efficient due to an increase in the role of higher order cyclotron harmonics.

[7] Another important issue is the dependence of wave amplitudes on the magnetic latitude. Pitch angle diffusion mainly takes place at intermediate and high latitudes, due to the dependence of the $g_{\lambda}(X)$ distribution on $\lambda$. Therefore, one should calculate the diffusion coefficients using a realistic dependence for $B_{w}(\lambda)$ (the effect of the $B_{w}$ variation on $\lambda$ was previously mentioned by Horne et al. [2005]). Moreover, the calculation of diffusion coefficients using the averaged value of $B_{w}$ for a given $\lambda$ cannot be considered as representative because spacecraft observations of the wave amplitude distribution $P_{\lambda}\left(B_{w}\right)$ clearly demonstrate a non-Gaussian form for $P_{\lambda}\left(B_{w}\right)$ with power law tails [Cully et al., 2008; Agapitov et al., 2011; Bunch et al., 2012]. Therefore, one should use $P_{\lambda}\left(B_{w}\right)$ to calculate the corresponding distribution of the diffusion coefficients, taking into account the dependence of $B_{w}$ and $g_{\lambda}(X)$ on $\lambda$.

\section{The Distribution of the Wave Amplitudes, $B_{w}$}

[8] For this work we utilized the data set for VLF waves, as observed by Cluster from March 2001 to December 2009, in an area located inside the radiation belt region (i.e. confined for the $\pm 45^{\circ}$ magnetic latitude and the $L$-shell $\left.\in[4,5]\right)$. The Cluster data set contains a sufficient number of measurements for performing a statistical study of the presented range of magnetic local times (MLT) and $L$-shells [Agapitov et al., 2011]. Our analysis was primarily based on data from the Spatiotemporal Analysis of Field Fluctuations - Spectrum Analyzer (STAFF-SA) experiment [Cornilleau-Wehrlin et al., 2003] that provides the complete spectral matrix (the real and the imaginary parts) of the three magnetic components as measured by the STAFF search coil magnetometer. The spectral matrix was computed on-board for 27 frequency channels that were logarithmically spaced between $8.8 \mathrm{~Hz}$ and $3.56 \mathrm{kHz}$. For the lower band chorus the full range of $L$-shells and magnetic latitudes was available. However, coverage by 5-7 channels was only available for $L>4$. The behavior of the upper band chorus frequency range could only be studied for $L>5.5$. Thus, chorus waves were mainly studied out of the plasmasphere. The sensitivity of STAFF search coil magnetometers was $5.10^{-3} \mathrm{nT} \mathrm{Hz}^{-1 / 2}$ at $1 \mathrm{~Hz}$, and $4.10^{-5} \mathrm{nT} \mathrm{Hz}{ }^{-1 / 2}$ at $100 \mathrm{~Hz}$ and $4 \mathrm{kHz}$ [Cornilleau-Wehrlin et al., 2003]. We excluded measurements that had amplitudes below double the STAFF-SA sensitivity level. The analyzed wave frequency range included electron whistler waves from the 0.1 electron cyclotron frequency $f_{c e}$ (approximately $1000 \mathrm{~Hz}$ for the processed $L$-shells) up to the electron cyclotron frequency $f_{c e}$. The range is known to be dominated by the lower-band chorus $\left(0.1 f_{c e}<f<0.5 f_{c e}\right)$, and by upper-band chorus waves $\left(0.5 f_{c e}<f<1.0 f_{c e}\right)$. Hiss waves can be observed above $0.1 f_{c e}$ but their intensity rapidly declines above $1 \mathrm{kHz}$ [Meredith et al., 2004].

[9] The global occurrence rate of whistler waves was presented by Agapitov et al. [2011]. The main portion of intense chorus waves was observed in the range from 06:00 to 14:00 MLT, and in the $L$-shell range from four to seven [Agapitov et al., 2011], for low, and intermediate and high geomagnetic activity, respectively. The major portion of large amplitude chorus waves was detected near local noon. In general, the distribution of large amplitude chorus waves becomes more uniform with increasing magnetic activity. To collect statistics for high geomagnetic activity, the following three geomagnetic activity regimes were processed: low $\left(K_{p}<3\right)$, intermediate $\left(3<K_{p}<5\right)$, and high $\left(K_{p}>5\right)$. We separated the entire range of the MLT into two sectors: a dawn/day sector that corresponds to MLT from 02:00 to 14:00, and a dusk/night sector that corresponds to a MLT from 14:00 to 02:00. The probability density functions (PDF) for the amplitudes of the wave magnetic fields are shown in Figure 1. The PDFs for the dawn/day and dusk/night sectors, and for low and high geomagnetic activity are also presented.

[10] Figure 1 shows large amplitudes for the chorus in the dawn/day sector for $\lambda>15^{\circ}$. The result seems to be consistent with the data presented by Meredith et al. [2001]. Bunch et al. [2012] also found results in agreement with Figure 1 for the wave intensity distribution with latitude on Polar spacecraft, with high amplitudes observed at high latitudes up to $45^{\circ}$. For $\lambda<15^{\circ}$ the wave amplitudes were lower and a local minimum in the intensity of the chorus was observed near the equator for the dawn/day sector, consistent with the results of Horne et al. [2005] obtained from CRRES measurements for the MLT from 06:00 to 12:00. Moreover, an increase in the wave intensity for the dusk/night sector for $\lambda<15^{\circ}$, shown in Figure 1 (large $K_{p}$ ), was also determined by Horne et al. [2005] for MLT from 23:00 to 06:00. Although, our statistics were, in general, consistent with CRESS data, such a comparison should be considered with care since the amplitudes of the wave magnetic field for CRESS spacecraft were obtained using a recalculation of the electric field amplitudes with a certain approximation for the wave-normal angle $\theta$ [see Horne et al., 2005; Ni et al., 2011]. As stated by Agapitov et al. [2011], 


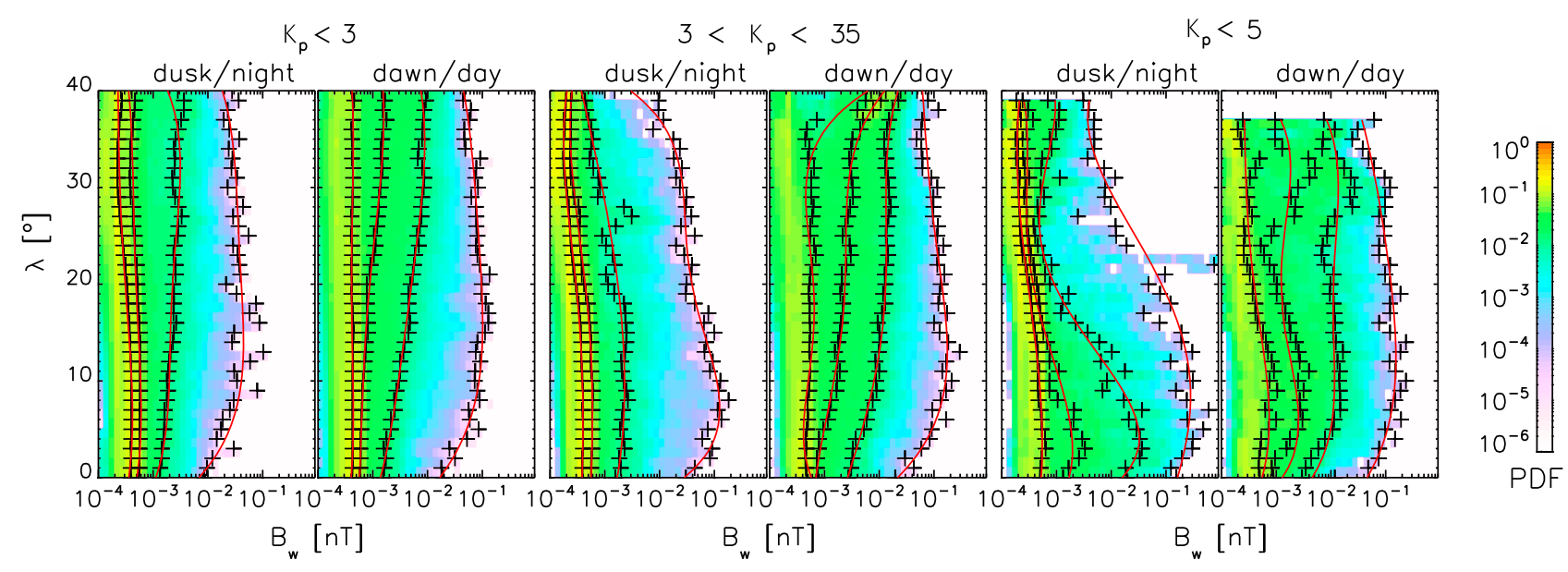

Figure 1. The distribution of the wave amplitudes $\left(B_{w}\right)$ for the dawn/day (MLT from 02:00 to 14:00) and dusk/night (MLT from 14:00 to 02:00) sectors. The cumulative distribution function levels of probabilities are equal to $0.5,0.75,0.95$, and 0.999 and are indicated by crosses. The fittings of these levels by the fifthorder polynomial on $\lambda$ are shown by lines.

$\theta$ substantially varies with $\lambda$. The effect should result in a variation of the ratio of the magnetic to electric field wave magnitudes.

[11] Figure 1 indicates that the distribution of the wave amplitudes $P_{\lambda}\left(B_{w}\right)$ depends on the magnetic latitude $\lambda$. To take into account this dependence and to obtain the distribution of the diffusion coefficients, we used the following approach. For each $\lambda$ we separated the range of $B_{w}$ variations, $B_{w} \in\left[B_{w}^{(\min )}, B_{w}^{(\max )}\right]$ into four intervals. The first portion corresponded to $5 \%$ of the most intense waves with amplitudes of $B_{w} \in\left[B_{w}^{(5 \%)}, B_{w}^{(\max )}\right]$. The second portion corresponded to the $15 \%$ of waves with the largest amplitudes, but smaller than $B_{w}^{(5 \%)}$ (for these waves we used $B_{w} \in$ $\left.\left[B_{w}^{(15 \%)}, B_{w}^{(5 \%)}\right]\right)$. The third portion corresponded to the $30 \%$ of waves with the largest amplitudes, but smaller than $B_{w}^{(15 \%)}$ (i.e. $\left.B_{w} \in\left[B_{w}^{(30 \%)}, B_{w}^{(15 \%)}\right]\right)$. The last portion corresponded to the remaining $50 \%$ of the distribution, for which $B_{w} \in\left[B_{w}^{(\mathrm{min})}, B_{w}^{(30 \%)}\right]$. A schematic view of this separation is presented in Figure 2. The PDF quantiles for each magnetic latitude, $\lambda$, are indicated by crosses in Figure 1. We approximated the dependence of the $B_{w}$ levels on $\lambda$ using the fifth-order polynomials (see Appendix C). These fittings are shown with red curves.

\section{Diffusion Coefficients}

[12] Calculation of the distribution of the coefficients of pitch angle diffusion $D_{\alpha \alpha}$ can be carried out in the framework of the approach described by Glauert and Horne [2005] using the distribution of wave amplitudes, $P_{\lambda}\left(B_{w}\right)$. However, several important modifications should be made to account for the variations of the main parameters for wave distributions with magnetic latitude (see Appendices A and $\mathrm{C})$.

[13] At each latitude $\lambda$, we calculated the local coefficients, $D_{\alpha \alpha}\left(\alpha, \lambda, B_{w}\right)$, accordingly to the scheme described in Appendix A. For the procedure, we used the Gaussian distribution for a wave-frequency $\hat{B}^{2}(\omega)$ and a variable $X=\tan \theta$, $g_{\lambda}(X) \sim \exp \left(-\left(X-X_{m}\right)^{2} / X_{w}^{2}\right)$. The distribution $\hat{B}^{2}(\omega)$ was assumed to be independent of the magnetic latitude. The characteristics of $\hat{B}^{2}(\omega)$ corresponded to lower band chorus waves (i.e. $\omega \in[0.125,0.575] \Omega_{e 0}$ ) with $\hat{B}^{2}(\omega)$ peaked around $0.35 \Omega_{e 0}$, where $\Omega_{e 0}=2 \pi f_{c e}$ is the electron gyrofrequency evaluated at the equator (see Appendix A). The dependence of parameters $X_{m}$ and $X_{w}$ on $\lambda$ was taken according to the observational data [Agapitov et al., 2011]. We used the polynomial fit of the functions $X_{m}, w(\lambda)$ [Artemyev et al., 2012] (see also Appendix A).

[14] We calculated the local diffusion coefficients for the five amplitudes $\left(B_{w}^{\min }, B_{w}^{30 \%}, B_{w}^{15 \%}, B_{w}^{5 \%}, B_{w}^{\max }\right)$ at each $\lambda$. Thus, we obtained four ranges of $D_{\alpha \alpha}$ values with their corresponding probabilities. Final values were obtained by

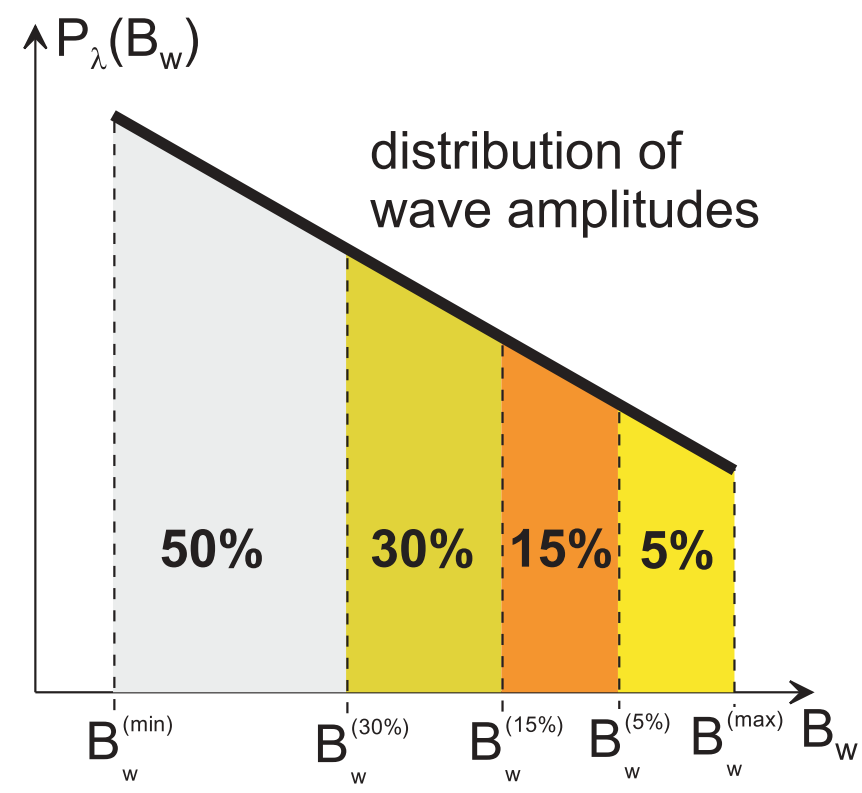

Figure 2. The scheme of the four part separation of the $B_{w}$ distribution. 
$<\mathrm{D}_{\alpha_{0} \alpha_{0}}>/ \mathrm{p}^{2}\left[\mathrm{~s}^{-1}\right]$ for MTL from 02:00 to $14: 00$
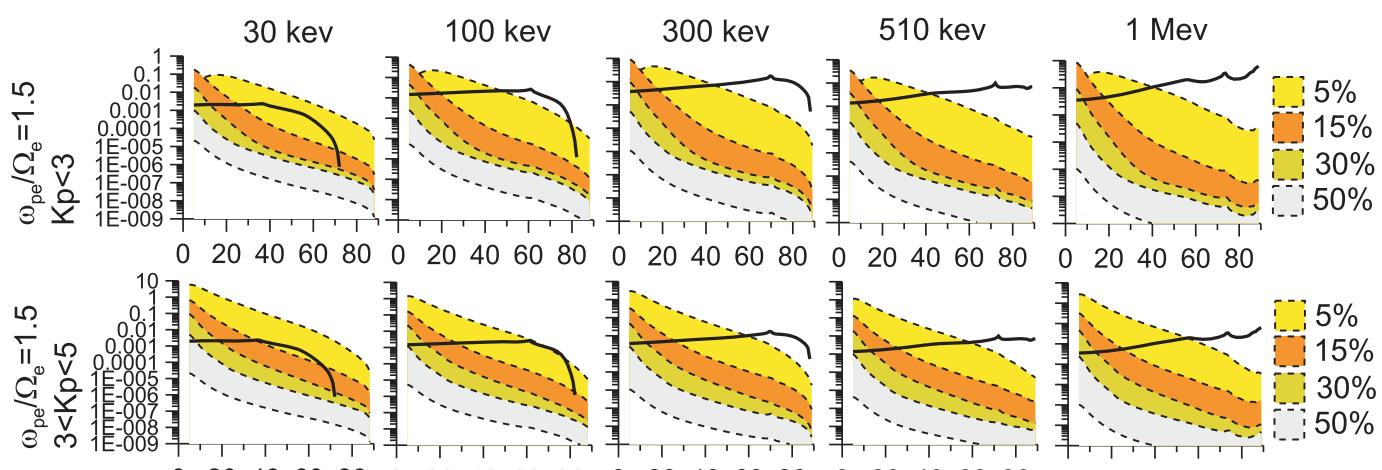

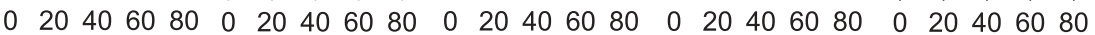

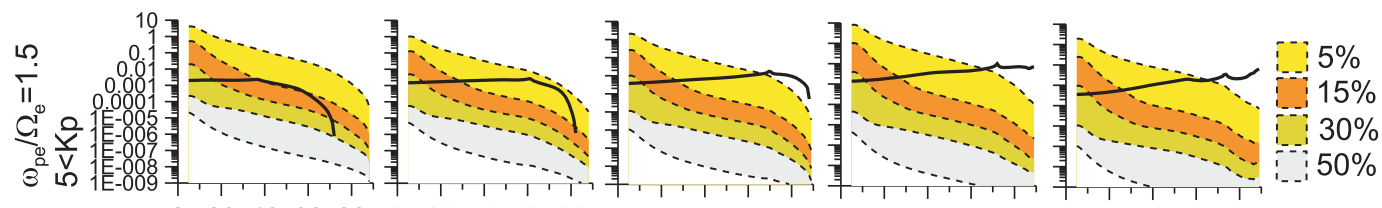

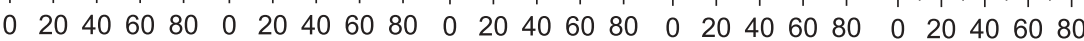
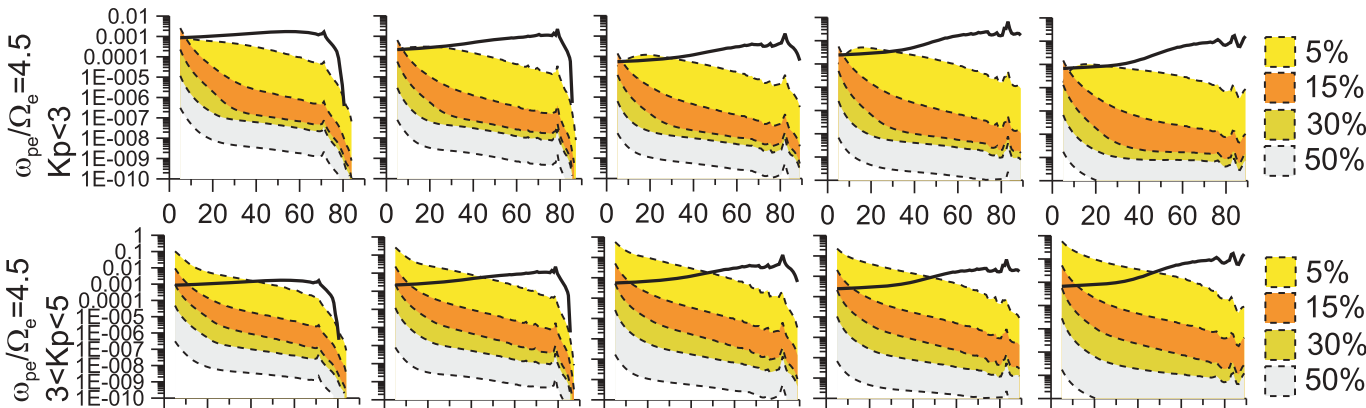

020406080
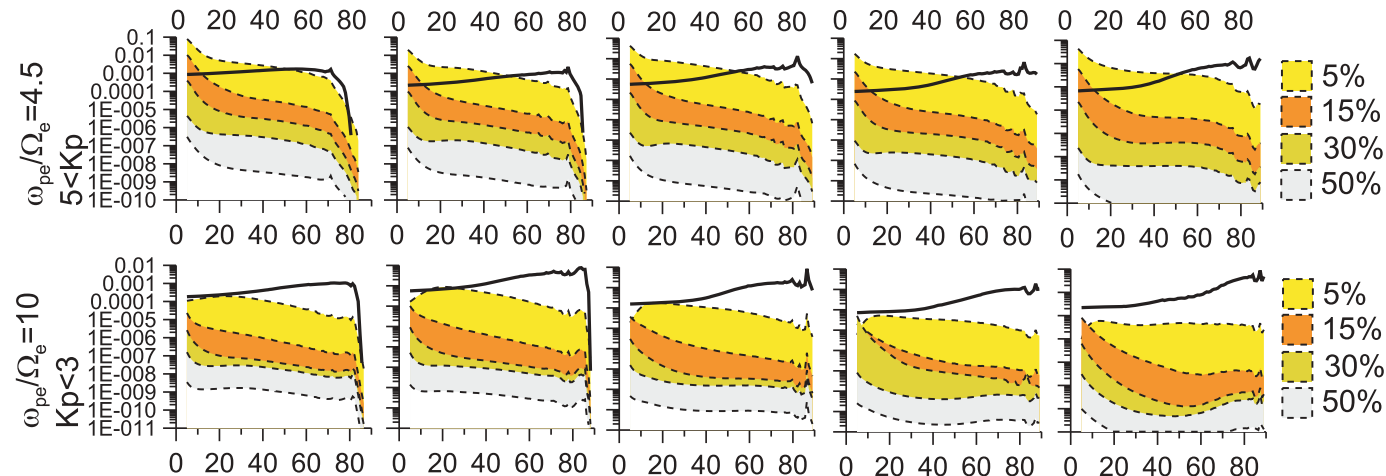

$020406080 \quad 020406080$
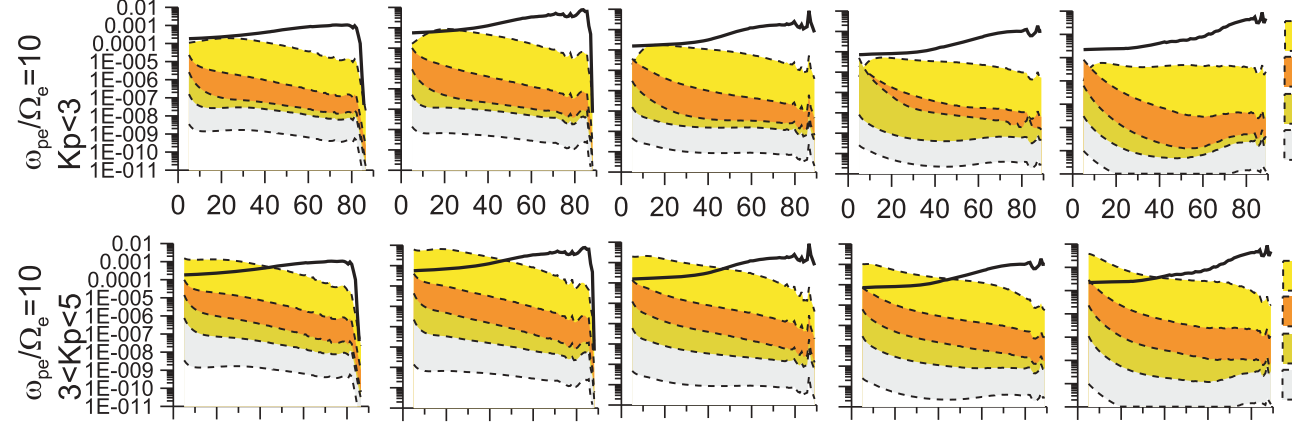

$5 \%$

$15 \%$

$30 \%$

$50 \%$
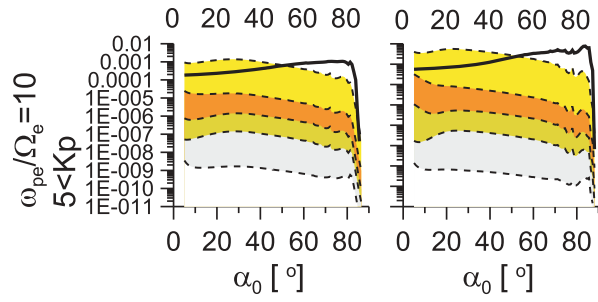

$020406080 \quad 020406080$
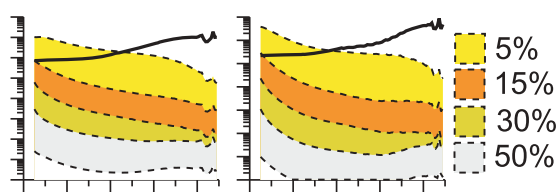

$20406080 \quad 0 \quad 20406080$

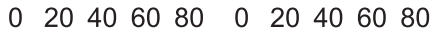

Figure 3. The distributions of $\left\langle D_{\alpha_{e q} \alpha_{e q}}\right\rangle$ for the dawn/day sector were calculated for five energies and three values of $\omega_{p e} / \Omega_{e}$ evaluated at the equator ( $\omega_{p e}$ is constant along field lines). Solid black curves show $\left\langle D_{\alpha_{e q} \alpha_{e q}}\right\rangle_{\text {const }}$ calculated with a constant wave amplitude $B_{w}=100 \mathrm{pT}$ and the constant parameters $X_{m}=0$, $X_{w} \stackrel{e q}{=} 0.577$. 
$<\mathrm{D}_{\alpha_{0} \alpha_{0}}>/ \mathrm{p}^{2}\left[\mathrm{~s}^{-1}\right]$ for MTL from 14:00 to 02:00
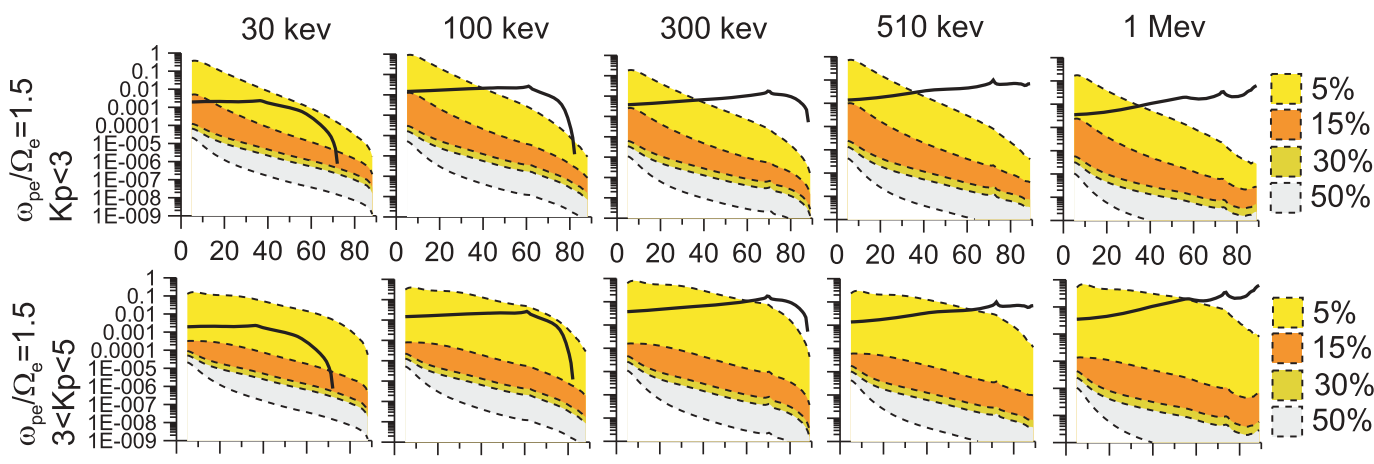

$020406080 \quad 0 \quad 20406080 \quad 0 \quad 20406080 \quad 0 \quad 20406080 \quad 0 \quad 20406080$

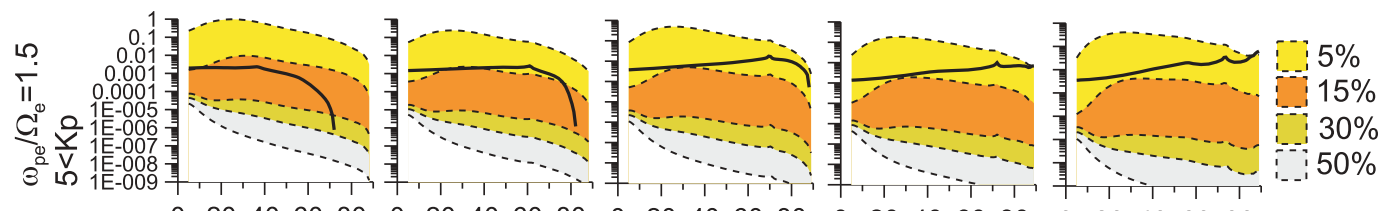

$020406080 \quad 0 \quad 20406080 \quad 0 \quad 20406080 \quad 0 \quad 204060 \quad 80 \quad 0 \quad 20406080$

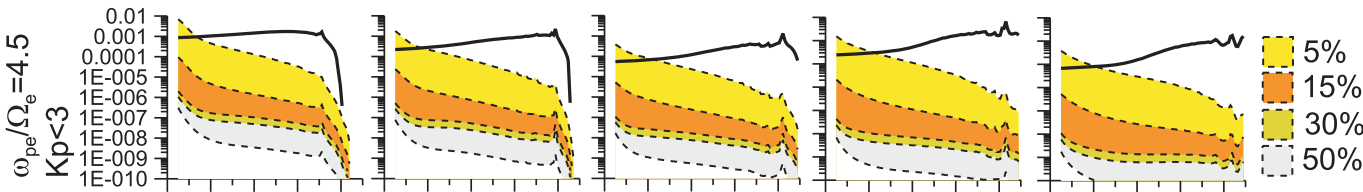

$020406080 \quad 0 \quad 20406080 \quad 0 \quad 20406080 \quad 0 \quad 20406080 \quad 0 \quad 20406080$
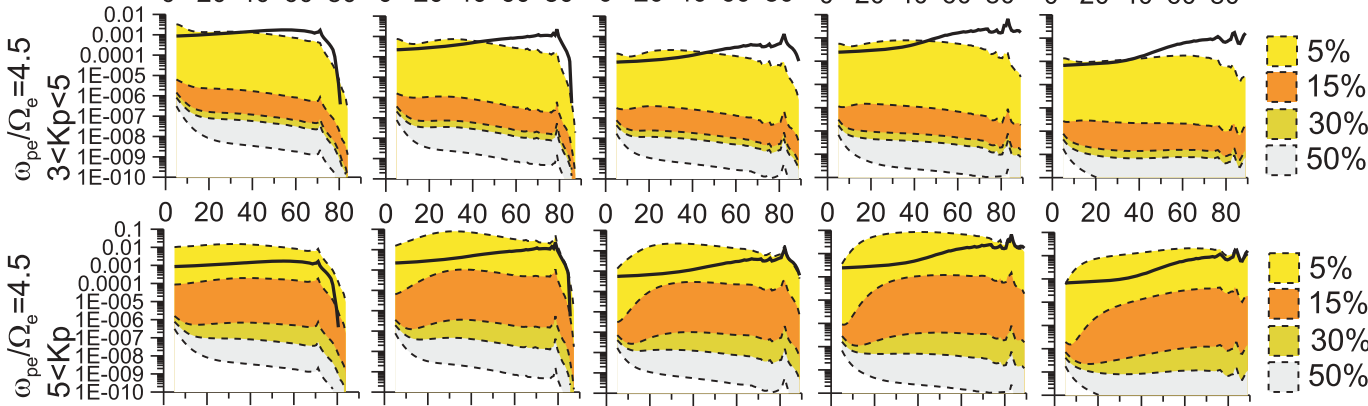

$020406080 \quad 0 \quad 20406080 \quad 0 \quad 204060 \quad 80 \quad 0 \quad 204060 \quad 80 \quad 0 \quad 20406080$
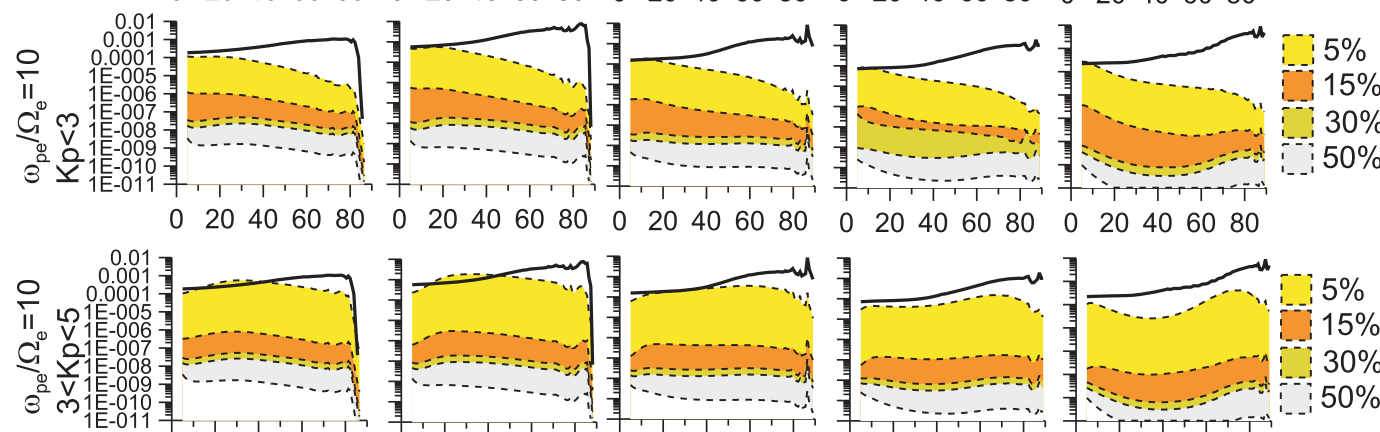

$020406080 \quad 020406080$
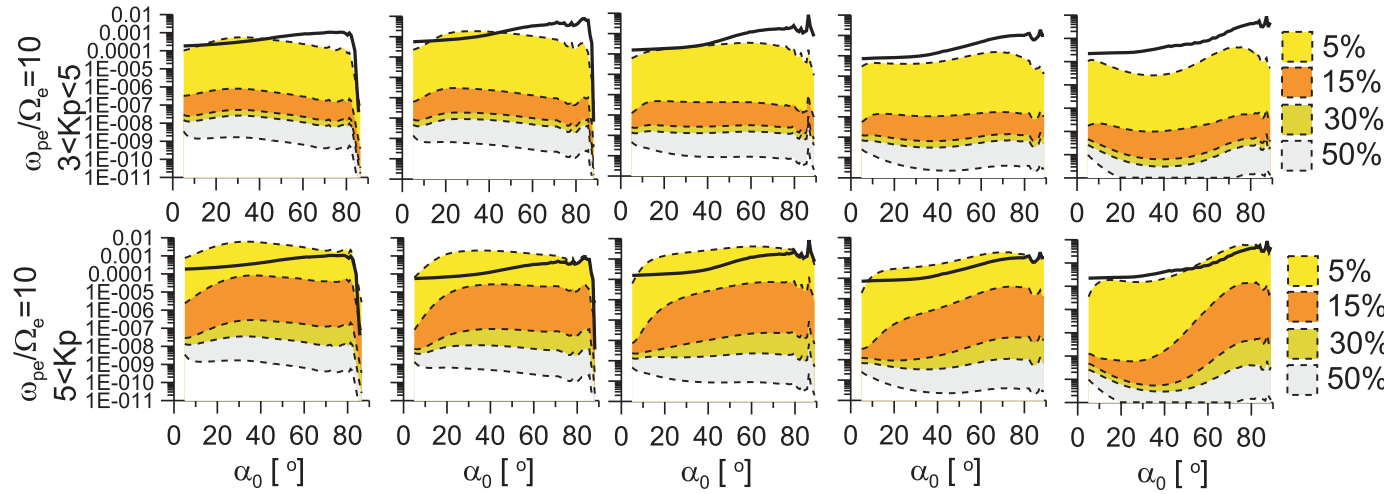

Figure 4. The distributions of $\left\langle D_{\alpha_{e q} \alpha_{e q}}\right\rangle$ for the dusk/night sector calculated for five energies and three values of $\omega_{p e} / \Omega_{e}$ evaluated at the equator ( $\omega_{p e}$ is constant along field lines). Solid black curve show $\left\langle D_{\alpha_{e q} \alpha_{e q}}\right\rangle_{c o n s t}$ calculated with a constant wave amplitude $B_{w}=100 \mathrm{pT}$ and constant parameters $X_{m}=0$, and $X_{w}=0.577$. 
averaging over magnetic latitudes (see Appendix C). As a result, we determined the distribution of $\left\langle D_{\alpha_{e q} \alpha_{e q}}\right\rangle$, where $\alpha_{e q}$ is the equatorial electron pitch angle.

[15] We used the wave amplitude distributions for two MLT sectors and three ranges of the $K_{p}$ index. Diffusion coefficients were calculated for five values of the electron energy $(30 \mathrm{keV}, 100 \mathrm{keV}, 300 \mathrm{keV}, 510 \mathrm{keV}$, and $1 \mathrm{MeV})$. We also calculated the diffusion coefficient $\left\langle D_{\alpha_{\text {eq }} \alpha_{e q}}\right\rangle_{\text {const }}$ for the constant value of the wave amplitude $B_{w}=100 \mathrm{pT}$, and for the constant values of the $X_{m, w}$ parameters taken from Glauert and Horne [2005], for comparison.

[16] Electron density determines the local value of the plasma frequency $\omega_{p e}$ and depends on the $L$-shell [Sheeley et al., 2001]. Due to the relatively weak variation of $\omega_{p e}$ along field lines [Reinisch et al., 2004; Horne et al., 2005] we calculated the diffusion coefficients with $\omega_{p e}=$ const for $\lambda<40^{\circ}$. The effect of a strong variation of the plasma frequency along field lines was considered by Artemyev et al. [2012], where a decrease in the pitch angle diffusion rates in the vicinity of a loss cone was determined for a $\omega_{p e}$ growing with $\lambda$. We used three values for the $\omega_{p e} / \Omega_{e 0}$ ratio $(1.5,4.5$, and 10). As shown by Meredith et al. [2003], the ratio $\omega_{p e} / \Omega_{e 0}$ can vary from 1.7 to 10 depending on geomagnetic activity and MLT, while the average value is around 3-4 (also see the statistics presented by Horne et al. [2005]). Thus, our calculations span almost the entire observed range of $\omega_{p e} / \Omega_{e 0}$ variations.

[17] The distributions of $\left\langle D_{\alpha_{e q} \alpha_{e q}}\right\rangle$ for the dawn/day sector are shown in Figure 3. One can see that for $K_{p}>3$ and a small $\alpha_{e q}$ diffusion coefficients can be much larger than those obtained with $B_{w}=100 \mathrm{pT}$ and $X_{m, w}=$ const. For $5 \%$ of the most intense waves we determined a pitch angle diffusion one-two orders of magnitude larger than $\left\langle D_{\alpha_{e q} \alpha_{e q}}\right\rangle_{\text {const }}$.

[18] The difference between the dependencies of $\left\langle D_{\alpha_{e q} \alpha_{e q}}\right\rangle$ and $\left\langle D_{\alpha_{e q} \alpha_{e q}}\right\rangle_{\text {const }}$ on $\alpha_{e q}$ (the dotted and solid curves in Figure 3) demonstrates the role of wave amplitude variation with magnetic latitude and the effect of the strong deviation of wave normals from the local magnetic field direction. While for small equatorial pitch angles we have $\left\langle D_{\alpha_{e q} \alpha_{e q}}\right\rangle>$ $\left\langle D_{\alpha_{e q} \alpha_{e q}}\right\rangle_{c o n s t}$, for particles with large equatorial pitch angles we obtained $\left\langle D_{\alpha_{e q} \alpha_{e q}}\right\rangle\left\langle\left\langle D_{\alpha_{e q} \alpha_{e q}}\right\rangle_{\text {const }}\right.$. Particles with $\alpha_{e q}>60^{\circ}$ only interact with resonance waves in the vicinity of the equatorial plane, whereas wave amplitudes have a local minimum (see Figure 1) and the mean value of the $\theta$ distribution is small [Agapitov et al., 2011].

[19] A comparison of $\left\langle D_{\alpha_{e q} \alpha_{e q}}\right\rangle$ calculated for $K_{p}>3$ and for $K_{p}<3$ exhibited differences in the wave amplitude distributions. The upper boundary of the wave amplitudes $B_{w}^{(\max )}$ (and, as a result, the upper boundary of the $\left\langle D_{\alpha_{e q} \alpha_{e q}}\right\rangle$ value) differed by one order of magnitude for small and large $K_{p}$ 's. Moreover, the $P_{\lambda}\left(B_{w}\right)$ distribution for a small $K_{p}$ had a much narrower tail in comparison with the one for a large $K_{p}$. As a result, the difference between $5 \%$ of the most intense waves and the next $15 \%$ was much larger for a small $K_{p}$.

[20] The distributions of $\left\langle D_{\alpha_{e q} \alpha_{e q}}\right\rangle$ for the dusk/night sector are shown in Figure 4. We used the same particle energies and values for the $\omega_{p e} / \Omega_{e 0}$ parameter as shown in Figure 3. Diffusion coefficients, calculated with the constant wave amplitude $B_{w}=100 \mathrm{pT}$, were comparable with the results obtained for the dusk/night sector for $5 \%$ of the most intense waves and $K_{p}>5$. In comparison, the dawn/day sector $\left\langle D_{\alpha_{e q} \alpha_{e q}}\right\rangle_{\text {const }}$ was located near the boundary between the $5 \%$ and $15 \%$ ranges or even inside the $15 \%$ range for $\alpha_{e q}<30^{\circ}$. The decrease of $\left\langle D_{\alpha_{e q} \alpha_{e q}}\right\rangle$ relative to $\left\langle D_{\alpha_{e q} \alpha_{e q}}\right\rangle_{\text {const }}$ corresponded to the decrease of wave amplitudes within the dusk/night sector in comparison to the dawn/day sector.

\section{Discussion}

[21] One of the important characteristics of chorus wave propagation is a rapid deviation of direction of propagation from the magnetic field direction. Here, this effect was taken into account in calculations of $\left\langle D_{\alpha_{e q} \alpha_{e q}}\right\rangle$. The strong deviation of the whistler wave normal from the local direction of the magnetic field at higher latitudes is a well-known effect that is obtained in various ray tracing models [Lyons and Williams, 1984; Boskova et al., 1990; Bortnik et al., 2011, and references therein]. However, only as a result of recent spacecraft missions such as Polar [Haque et al., 2010], Cluster [Santolik et al., 2009; Agapitov et al., 2011], and THEMIS [ Li et al., 2011] has this effect been statistically determined. The obtained distribution of $\theta$ demonstrates rapid growth of the mean value $X_{m}$ and the variance $X_{w}$ with $\lambda$ [Agapitov et al., 2011]. Large values of $\theta$ lead to an increase in the role of higher order cyclotron resonances for particles with small equatorial pitch angles (see the review by Shklyar and Matsumoto [2009, and references therein]). The effect results in a significant increase of $\left\langle D_{\alpha_{e q} \alpha_{e q}}\right\rangle$ [see Shprits and Ni, 2009; Ni et al., 2011; Artemyev et al., 2012]. As a result, we obtained a $\left\langle D_{a_{e q} \alpha_{e q}}\right\rangle$ larger than $\left\langle D_{\alpha_{e q} \alpha_{e q}}\right\rangle_{\text {const }}$ for small $\alpha_{e q}$, while the observed wave amplitudes were usually less than $100 \mathrm{pT}$.

[22] Although, we obtained a strong increase of $\left\langle D_{\alpha_{e q} \alpha_{e q}}\right\rangle$ due to a $X_{m},{ }_{w}$ increase with $\lambda$, we expect a weaker effect for energy diffusion coefficients as well as for the coefficients describing mixed diffusion. An increase in $\left\langle D_{\alpha_{e q} \alpha_{e q}}\right\rangle$ was provided due to the effect of higher cyclotron harmonics. The energy diffusion partial rates corresponding to certain harmonic numbers $n$ are $\left\langle D_{E E}^{n}\right\rangle \sim\left\langle D_{\alpha_{e q} \alpha_{e q}}^{n}\right\rangle / n^{2}$ [see, e.g., Lyons and Williams, 1984; Glauert and Horne, 2005]. Therefore, higher cyclotron harmonics would be suppressed by a factor of $1 / n^{2}$. However, we still could obtain intensification in energy diffusion within the vicinity of the loss cone, where electrons with small pitch angles reach higher latitudes (a significant increase in $\left\langle D_{E E}^{n}\right\rangle$ for $X_{m} \sim 1$ was previously shown by Shprits and $N i$ [2009]). An investigation of the effect of an $X_{m, w}$ increase with $\lambda$ on energy diffusion will be the subject of a future publication.

[23] We used the distribution of the wave amplitude $P_{\lambda}\left(B_{w}\right)$ obtained from direct spacecraft observations, an important improvement due to the effect of $\theta$ deviations from zero to relatively large values at high latitudes. If $\theta$ is close to the value at the resonant cone, the electrostatic component of whistler waves becomes very important [Ginzburg and Rukhadze, 1975; Sazhin and Horne, 1990]. Therefore, the description of wave-particle resonant interactions with electromagnetic waves of a constant amplitude (independent of $\lambda$ ) cannot be applied. We used the distribution of the wave magnetic field amplitudes $B_{w}$, and, as a result, our calculations include the effect of wave amplitude variation with $\theta$ along the trace of wave propagation from the equator to high latitudes. 
[24] The obtained intensification of pitch angle diffusion due to a $X_{m, w}$ increase with $\lambda$ should lead to a decrease in the lifetime of trapped electrons in radiation belts. The lifetimes can be roughly estimated as $\tau \sim 1 /\left\langle D_{a_{e q} \alpha_{e q}}\right\rangle$, where $\left\langle D_{\alpha_{e q} \alpha_{e q}}\right\rangle$ is evaluated in the vicinity of a minimum of $\left\langle D_{\alpha_{e q} \alpha_{e q}}\right\rangle \tan \alpha_{e q}$ [Albert and Shprits, 2009]. Therefore, the obtained increase of $\left\langle D_{\alpha_{e q} \alpha_{e q}}\right\rangle$ by one-two orders of magnitude for $5 \%$ of the most intense waves results in a substantial decrease of $\tau$. Moreover, due to the effect of $X_{m}$, ${ }_{w}$ dependencies on $\lambda$, one can determine a significant decrease in $\tau$ even for $15 \%-20 \%$ of the waves with the largest amplitudes. Analytical estimates of this decrease, as shown by Mourenas et al. [2012], can be approximately one order of magnitude in comparison with the lifetime calculated for parallel whistler waves propagation [Shprits et al., 2007]. Therefore, a strong decrease in lifetime for $20 \%$ of the probability distribution indicates that the main particle loss could correspond to the sporadic intensification of wave activity, rather than to slow permanent diffusion. In future work, such a conclusion should be proven by direct numerical solutions of the Fokker-Plank equation. In this work, we considered a wide range of $K_{p}$ indices, the MLT, and magnetic latitudes up to $40^{\circ}$, while restricting our study only to $L$-shell $\in[4,5]$ where Cluster provided broad statistics. On the other hand, information regarding the dependence of the diffusion rates on $L$-shells is important for studying electron injections into the radiation belt from the magnetotail. Such injections often propagate up to $L$-shells 7-8 (see, e.g., statistics by Dubyagin et al. [2011, and references therein]) or even deeper (injections observed inside the geostationary orbit were discussed by Friedel and Korth [1996] and Ingraham et al. [2001]). Further electron transport toward the outer radiation belt is provided by radial diffusion, while some portions of the electron population could be scattered into the loss cone during this slow process. Estimates for the surviving electron population to reach the outer radiation belt require calculations of pitch angle diffusion rates, as a function of the $L$-shell. The coefficient $\left\langle D_{\alpha \alpha}\right\rangle$ depends on the $L$-shell due to a variation of the background magnetic field and $\omega_{p e}$ with $L$ (estimates of these dependencies can be found in) [Shprits et al., 2007; Mourenas et al., 2012]. The second effect determining particle losses corresponds to the deviation of field line geometry from the dipolar configuration with increases of L [Orlova and Shprits, 2010; Ni et al., 2011]. Therefore, in order to obtain $\left\langle D_{\alpha \alpha}\right\rangle$ as function of $L$, one needs to take into account the combination of the effects of the nondipolar magnetic field and the dependencies of the main parameters on the $L$-shell. We will address these issues in future studies.

\section{Conclusions}

[25] In this work, we calculated the distribution of pitch angle diffusion coefficients based on the statistical distribution of the wave amplitudes $P_{\lambda}\left(B_{w}\right)$ and the distribution of the wave normal directions $g_{\lambda}(X)$ obtained from Cluster observations. We showed that the increase of the $\theta$ angle with magnetic latitude $\lambda$ leads to a significant increase of $\left\langle D_{\alpha_{e q} \alpha_{e q}}\right\rangle$, in agreement with previous work [Shprits and $\mathrm{Ni}$, 2009; Artemyev et al., 2012]. We demonstrated that for small equatorial pitch angles the pitch angle diffusion could be substantially larger than the one calculated for
$B_{w}=100 \mathrm{pT}$ and $X_{m}=0$. The effect is stronger for the dawn/day sector and for a large $K_{p}$. Our results clearly show that the dependence of $\left\langle D_{\alpha_{e q} \alpha_{e q}}\right\rangle$ on the equatorial pitch angle $\alpha_{e q}$ substantially differs for the realistic dependence of $B_{w}$ and $X=\tan \theta$ on $\lambda$, in comparison with previous calculations where the approximation $X_{m, w}, B_{w}=$ const was applied. In particular, values of the pitch angle diffusion coefficient for small values of $\alpha_{e q}$ are significantly larger, while the diffusion for electrons with $\alpha_{e q}>60^{\circ}$ is significantly slower than previous estimates for $X_{m},{ }_{w}, B_{w}=$ const.

\section{Appendix A: Calculation of the Local Diffusion Coefficients}

[26] To calculate the local diffusion coefficients for a given $\lambda$ we used the following expression [see Glauert and Horne, 2005]:

$$
D_{\alpha \alpha}=\frac{e^{2}}{4 \pi} \sum_{i, n} \int_{X_{\min }}^{X_{\max }} \frac{G \hat{B}^{2}\left|\Phi_{n}\right|^{2} X d X}{1+X^{2}} \frac{\left|\frac{n \Omega_{e}}{\gamma}-\omega_{i, n} \sin ^{2} \alpha\right|^{2}}{\cos ^{2} \alpha\left|v_{\|}-\frac{\partial \omega}{\partial k_{\|}}\right|}
$$

where $\gamma$ is a relativistic factor; $v_{\|}=c \sqrt{1-\gamma^{-2}} \cos \alpha$ where $c$ is the speed of light; $n$ is a number of the harmonic; $i$ is the number of a resonant root for a given $n$; and the term $\left|\Phi_{n}\right|^{2}$ can be found in [Lyons, 1974; Lyons and Williams, 1984; Glauert and Horne, 2005]. The functions $\hat{B} 2\left(\omega_{i, n}\right)$ and $G=G\left(\omega_{i, n}\right)$ are defined as follows:

$$
\begin{aligned}
G & =2 \pi^{2} g_{\lambda}(X) /\left.\int_{X_{\min }}^{X_{\max }} \frac{g_{\lambda}(X) X}{\left(1+X^{2}\right)^{3 / 2}} k_{i, n}^{2} \frac{\partial k}{\partial \omega}\right|_{X} d X \\
\hat{B}^{2} & =A \exp \left(-\left(\frac{\omega-\omega_{m}}{\delta \omega}\right)^{2}\right), \omega \in\left[\omega_{-}, \omega_{+}\right]
\end{aligned}
$$

and $\hat{B}^{2}=0$ if $\omega \notin\left[\omega_{-}, \omega_{+}\right]$, where $\omega_{ \pm}=\omega_{m} \pm \Delta \omega$. The constant $A$ is determined from the normalization $\int_{\omega}^{\omega_{+}} \hat{B}^{2}(\omega) d \omega=B_{w}^{2}$. The resonant wave number $k_{i, n}$ can be found from the dispersion relation $\omega\left(k_{i, n}\right)=\omega_{i, n}$, and the resonant condition $\omega_{i, n}-k_{i, n} \cos \theta v_{\|}=-n \Omega_{e} / \gamma$ (see Appendix B). The electron gyrofrequency is $\Omega_{e}=|e| B(\lambda) /$ $m_{e} c=2 \pi f_{c e}$, where the magnetic field is defined according to the dipole model, as follows: $B(\lambda)=B_{e q} \sqrt{1+3 \sin ^{2} \lambda} /$ $\cos ^{6} \lambda$, and $B_{e q}$ is the equatorial value (in this paper we use $B_{e q}$ for $\left.L=4.5\right)$. Although the effect related to the deviation of $B(\lambda)$ from the dipole model can be important for $L$-shell $>7$ in the night side [Orlova and Shprits, 2010; Ni et al., 2011], here, we are mainly interested inthe $L$-shell $\sim 4.5$, where the dipole approximation can be safely used. We used $\omega_{m}=0.35 \Omega_{e 0}, \delta \omega=0.15 \Omega_{e 0}$, and $\Delta \omega=1.5 \delta \omega$, where $\Omega_{e 0}$ is the equatorial value of $\Omega_{e}$.

[27] We used a Gaussian approximation for the distribution of $X=\tan \theta$ :

$$
g_{\lambda}(X)=\exp \left(-\frac{\left(X-X_{m}\right)^{2}}{X_{w}^{2}}\right)
$$


where the mean value $X_{m}$ and the variance $X_{w}$ are functions of $\lambda$. We approximated the dependencies $X_{m, w}(\lambda)$ using polynomials (see a comparison of this approximation with experimental data in Artemyev et al. [2012]):

$$
\begin{aligned}
& X_{m}=0.79-0.36 l+0.11 l^{2}+0.16 l^{3} \\
& X_{w}=\sqrt{2}\left(0.84-0.4 l+2.0 l^{2}-1.1 l^{3}+0.17 l^{4}\right)
\end{aligned}
$$

where $l=\lambda / 10^{\circ}$ and $\lambda<40^{\circ}$.

\section{Appendix B: Resonant Frequencies}

[28] To calculate the frequencies and the corresponding wave numbers of the resonant wave-particle interaction one needs to solve a system consisting of a dispersion relation, $\omega$ $=\omega(k)$, and the resonant condition. In this Appendix, we derived the dispersion relation for low frequency $\left(\omega^{2} \ll \Omega_{e}^{2}\right)$ whistler waves. For systems with $\omega_{p e}^{2} / \Omega_{e}^{2} \gg 1$, where $\omega_{p e}$ is the plasma frequency one can write the simplified dispersion relation, as follows [see Ginzburg and Rukhadze, 1975]:

$$
\omega=\Omega_{e}|\cos \theta| \frac{k^{2} c^{2}}{k^{2} c^{2}+\omega_{p e}^{2}}
$$

[29] The relation is valid until $\omega \gg \omega_{L H}$, where $\omega_{L H}$ is the lower hybrid frequency. However, in this work we were interested in a wide range of ratios for $\omega_{p e} / \Omega_{e}$ (for example, we used $\left.\omega_{p e} / \Omega_{e} \in[1.5,10]\right)$. To take into account moderate values of $\omega_{p e} / \Omega_{e}$, we derived a new dispersion relation.

[30] We began with the dielectric tensor $\hat{\varepsilon}$, as follows:

$$
\hat{\varepsilon}=\left(\begin{array}{ccc}
\varepsilon_{1} & i \varepsilon_{2} & 0 \\
-i \varepsilon_{2} & \varepsilon_{1} & 0 \\
0 & 0 & \varepsilon_{3}
\end{array}\right)
$$

where $\varepsilon_{1}=1-\frac{\omega_{p e}^{2}}{\omega^{2}-\Omega_{e}^{2}}, \quad \varepsilon_{2}=\frac{\Omega_{e}}{\omega} \frac{\omega_{p e}^{2}}{\omega^{2}-\Omega_{e}^{2}}$, and $\varepsilon_{3}=$ $1-\frac{\omega_{p e}^{2}}{\omega^{2}}$. The dispersion relation can be obtained as a solution of equation $A_{1}+A_{2} \frac{\omega^{2}}{k^{2} c^{2}}+A_{3} \frac{\omega^{4}}{k^{4} c^{4}}=0$, where

$$
\begin{aligned}
& A_{1}=\varepsilon_{1} \sin ^{2} \theta+\varepsilon_{3} \cos ^{2} \theta \\
& A_{2}=-\varepsilon_{1} \varepsilon_{3}\left(1+\cos ^{2} \theta\right)-\left(\varepsilon_{1}^{2}-\varepsilon_{2}^{2}\right) \sin ^{2} \theta . \\
& A_{3}=\varepsilon_{3}\left(\varepsilon_{1}^{2}-\varepsilon_{2}^{2}\right)
\end{aligned}
$$

[31] The approximations $\varepsilon_{1} \approx-\frac{\omega_{p e}^{2}}{\omega^{2}-\Omega_{e}^{2}}, \varepsilon_{2} \approx \frac{\Omega_{e}}{\omega} \frac{\omega_{p e}^{2}}{\omega^{2}-\Omega_{e}^{2}}$, and $\varepsilon_{3} \approx-\frac{\omega_{p e}^{2}}{\omega^{2}}$ led to dispersion relation (B1). Here, we used $\varepsilon_{1} \approx-\frac{\omega_{p e}^{2}+\Omega_{e}^{2}}{\omega^{2}-\Omega_{e}^{2}}$ for the first term (we kept the term $\Omega_{e}^{2} / \omega_{p e}^{2}$ ). For this case we obtained the following dispersion relation:

$$
\omega=\Omega_{e}|\cos \theta| \frac{c^{2} k^{2}}{\sqrt{A_{4}^{2}+\frac{\Omega_{e}^{2}}{\omega_{p e}^{2}} c^{2} k^{2} A_{4}}}
$$

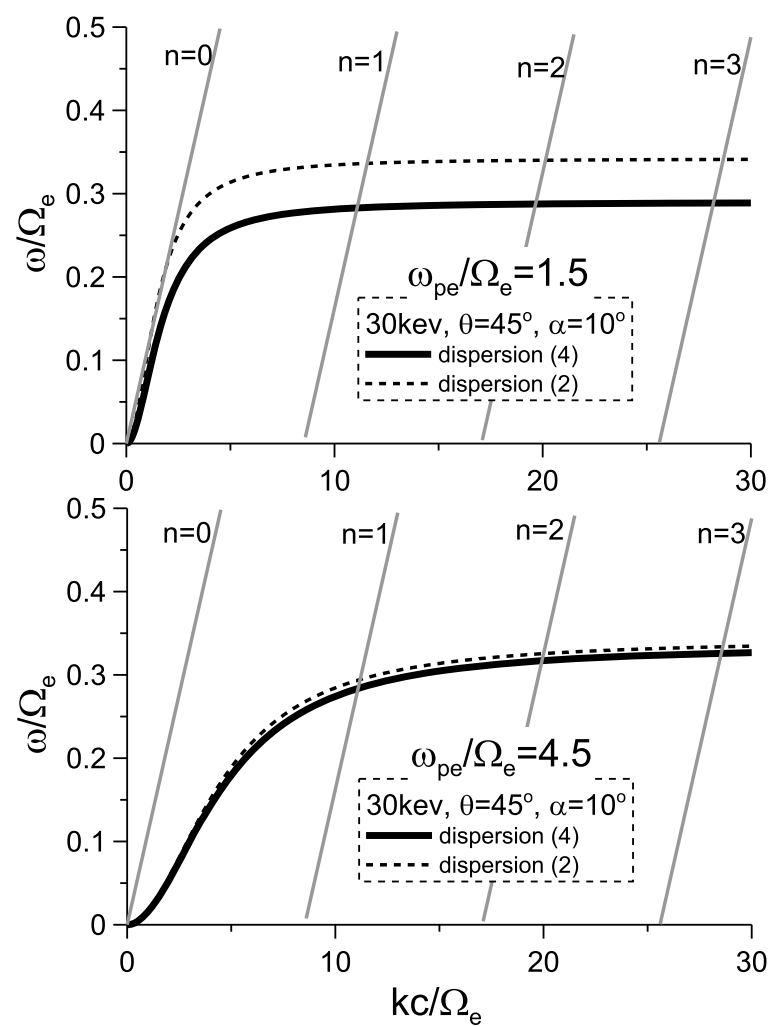

Figure B1. The dispersion relationships (B1) and (B3), and the resonant conditions are shown for two values of the ratio $\omega_{p e} / \Omega_{e}$.

where $A_{4}=c^{2} k^{2}+\omega_{p e}^{2}$. If we assume that $\omega_{p e}^{2} / \Omega_{e}^{2} \gg 1$, the dispersion relation (B3) can easily be reduced to equation (B1).

[32] The resonant condition for several $n$ 's and both dispersion relations $(\mathrm{B} 3, \mathrm{~B} 1)$ are presented in Figure B1. As one can see, some of the differences in the resonant frequencies for (B1) and (B3) can only be found for the ratio $\omega_{p e} / \Omega_{e} \sim 1$.

\section{Appendix C: Bounce Averaging}

[33] To obtain the averaged value of diffusion coefficients depending on the equatorial value of the pitch angle $\alpha_{e q}$, we used the following scheme. We calculated the local values of the diffusion coefficients for a given magnetic latitude $\lambda$ and the pitch angle $\alpha$, where $\sin ^{2} \alpha=\left(B(\lambda) / B_{e q}\right) \sin ^{2} \alpha_{e q}$. We then integrated over latitude, as follows:

$$
\left\langle D_{\alpha_{e q} \alpha_{e q}}\right\rangle=\frac{1}{T\left(\alpha_{e q}\right)} \int_{0}^{\lambda_{\mathrm{m}}} D_{\alpha \alpha}\left(\alpha_{e q}, \lambda\right) \frac{\cos \alpha \cos ^{7} \lambda}{\cos ^{2} \alpha_{e q}} d \lambda
$$

where the period of bounce oscillations is $T\left(\alpha_{e q}\right)=$ $1.30-0.56 \sin \alpha_{e q}$ [Hamlin et al., 1961]. The upper limit of the integration $\lambda_{m}$ is defined as $\lambda_{m}=\min \left(\lambda_{\max }, 40^{\circ}\right)$, where $\lambda_{\max }$ is the latitude of the mirror points (i.e. $\left(B\left(\lambda_{\max }\right) / B_{e q}\right)$ $\left.\sin ^{2} \alpha_{e q}=1\right)$. The local diffusion coefficients, $D_{\alpha \alpha}$, depend on $\lambda$ due to the following four effects:

[34] 1. The pitch angle is $\alpha=\alpha\left(\alpha_{e q}, \lambda\right)$. 
Table C1. The List of $c_{n}$ Coefficients for Two Sectors and Three $K_{p}$ Ranges $^{\mathrm{a}}$

\begin{tabular}{|c|c|c|c|c|c|c|}
\hline$B_{w}^{*}$ & $c_{0}$ & $c_{1}$ & $c_{2}$ & $c_{3}$ & $c_{4}$ & $c_{5}$ \\
\hline \multicolumn{7}{|c|}{ Dawn/day sector, $K_{p}>5$} \\
\hline$B_{w}^{\max }$ & -1.701 & 2.043 & -1.864 & 0.656 & 0.550 & -0.081 \\
\hline$B_{w}^{5 \%}$ & -2.541 & 1.287 & -0.269 & -0.714 & 0.418 & -0.062 \\
\hline$B_{w}^{15 \%}$ & -3.048 & 0.828 & 1.094 & -2.018 & 0.875 & -0.115 \\
\hline$B_{w}^{30 \%}$ & -3.379 & 0.197 & 1.498 & -1.798 & 0.661 & -0.078 \\
\hline \multicolumn{7}{|c|}{ Dawn/day sector, $5>K_{p}>3$} \\
\hline$B_{w}^{\max }$ & -1.854 & 1.391 & -0.776 & 0.191 & -0.020 & 0.0 \\
\hline$B_{w}^{5 \%}$ & -2.520 & 0.546 & -0.101 & -0.009 & 0.003 & 0.0 \\
\hline$B_{w}^{15 \%}$ & -3.103 & 0.096 & 0.480 & -0.270 & 0.041 & 0.0 \\
\hline$B_{w}^{30 \%}$ & -3.286 & -0.497 & 0.884 & -0.411 & 0.059 & 0.0 \\
\hline \multicolumn{7}{|c|}{ Dawn/day sector, $K_{p}<3$} \\
\hline$B_{w}^{\max }$ & -1.859 & 0.329 & 0.215 & -0.137 & 0.0002 & 0.0 \\
\hline$B_{w}^{5 \%}$ & -2.822 & 0.328 & -0.104 & 0.056 & -0.0097 & 0.0 \\
\hline$B_{w}^{15 \%}$ & -3.245 & 0.142 & -0.199 & 0.118 & -0.016 & 0.0 \\
\hline$B_{w}^{30 \%}$ & -3.390 & -0.009 & 0.079 & -0.061 & 0.012 & 0.0 \\
\hline \multicolumn{7}{|c|}{ Dusk/night sector, $K_{p}>5$} \\
\hline$B_{w}^{\max }$ & -0.798 & 0.726 & -0.837 & 0.259 & -0.037 & 0.0 \\
\hline$B_{w}^{5 \%}$ & -1.694 & 1.633 & -2.904 & 1.151 & -1.420 & 0.0 \\
\hline$B_{w}^{15 \%}$ & -2.740 & 0.241 & -0.833 & 0.367 & -0.049 & 0.0 \\
\hline$B_{w}^{30 \%}$ & -3.297 & 0.174 & -0.306 & 0.106 & -0.013 & 0.0 \\
\hline \multicolumn{7}{|c|}{ Dusk/night sector, $5>K_{p}>3$} \\
\hline$B_{w}^{\max }$ & -1.672 & 2.066 & -2.352 & 0.906 & -0.119 & 0.0 \\
\hline$B_{w}^{5 \%}$ & -2.706 & -0.047 & 0.338 & -0.235 & 0.0352 & 0.0 \\
\hline$B_{w}^{15 \%}$ & -3.241 & 0.048 & 0.012 & -0.053 & 0.0098 & 0.0 \\
\hline$B_{w}^{30 \%}$ & -3.394 & 0.061 & -0.027 & -0.030 & 0.0064 & 0.0 \\
\hline \multicolumn{7}{|c|}{ Dusk/night sector, $K_{p}<3$} \\
\hline$B_{w}^{\max }$ & -2.402 & 1.314 & -0.688 & 0.169 & -0.019 & 0.0 \\
\hline$B_{w}^{5 \%}$ & -2.896 & 0.299 & -0.067 & 0.020 & -0.0068 & 0.0 \\
\hline$B_{w}^{15 \%}$ & -3.266 & -0.052 & 0.147 & -0.090 & 0.0123 & 0.0 \\
\hline$B_{w}^{30 \%}$ & -3.431 & -0.009 & 0.071 & -0.058 & 0.0087 & 0.0 \\
\hline
\end{tabular}

${ }^{a}$ We also used $\log _{10} B_{w}^{\min }=-2.5$.

[35] 2. The resonant frequency $\omega_{i, n}$ depends on the local value of the gyrofrequency $\Omega_{e}=\Omega_{e}(\lambda)$ and the plasma frequency $\omega_{p e}=\omega_{p e}(\lambda)$ (in this paper we used the approximation $\omega_{p e}=$ const $)$.

[36] 3. The distribution of angles for the wave propagation $g_{\lambda}(X)$ depends on the magnetic latitude.

[37] 4. The local diffusion coefficient is proportional to the wave amplitude $D_{\alpha \alpha} \sim B_{w}^{2}$. We used the relationship $B_{w}=B_{w}(\lambda)$.

[38] First, two effects were taken into account for the majority of the calculations for the diffusion coefficients [Glauert and Horne, 2005; Summers et al., 2007; Albert, 2007, and references therein]. The effect of the $g_{\lambda}(X)$ dependence on the magnetic latitude was considered by Artemyev et al. [2012]. The effect of the $B_{w}$ variation with $\lambda$ is considered, for the first time, in this work.

[39] To take into account the dependence of $B_{w}$ on the magnetic latitude, we approximated $B_{w}(\lambda)$ using polynomials for five levels of probability (see the description above and the scheme in Figure 2).

$$
\log _{10} B_{w}=\sum_{n} c_{n} l^{n}
$$

where $l=\lambda / 10^{\circ}, B_{w}$ is measured in nT, and the coefficients $\left(c_{n}\right)$ are presented in Table $\mathrm{C} 1$. For comparison, we also calculated the diffusion coefficients for parallel whistler wave propagation. We used $X_{m}=0, X_{w}=0.577$, and $\lambda_{m}=$ $\lambda_{\max }$ according to Glauert and Horne [2005].

[40] Acknowledgments. We acknowledge useful discussions with D. Mourenas. This work was supported by CNES through Modeles d'ondes and Cluster Co-I DWP grants. The work of A.O. was supported by the STUDIUM program of the Region Centre in France. We thank the ESA Cluster Active Archive for providing the STAFF-SA data set.

[41] Robert Lysak thanks the reviewers for their assistance in evaluating this paper.

\section{References}

Agapitov, O., V. Krasnoselskikh, Y. V. Khotyaintsev, and G. Rolland (2011), A statistical study of the propagation characteristics of whistler waves observed by Cluster, Geophys. Res. Lett., 38, L20103, doi:10.1029/ 2011GL049597.

Albert, J. M. (2007), Simple approximations of quasi-linear diffusion coefficients, J. Geophys. Res., 112, A12202, doi:10.1029/2007JA012551.

Albert, J. M. (2008), Efficient approximations of quasi-linear diffusion coefficients in the radiation belts, J. Geophys. Res., 113, A06208, doi:10.1029/2007JA012936.

Albert, J. M., and Y. Y. Shprits (2009), Estimates of lifetimes against pitch angle diffusion, J. Atmos. Sol. Terr. Phys., 71, 1647-1652, doi:10.1016/ j.jastp.2008.07.004

Albert, J. M., N. P. Meredith, and R. B. Horne (2009), Three-dimensional diffusion simulation of outer radiation belt electrons during the 9 October 1990 magnetic storm, J. Geophys. Res., 114, A09214, doi:10.1029/ 2009JA014336.

André, R., F. Lefeuvre, F. Simonet, and U. S. Inan (2002), A first approach to model the low-frequency wave activity in the plasmasphere, Ann. Geophys., 20, 981-996, doi:10.5194/angeo-20-981-2002.

Artemyev, A., O. Agapitov, H. Breuillard, V. Krasnoselskikh, and G. Rolland (2012), Electron pitch-angle diffusion in the radiation belts: effects of whistler waves oblique propagation, Geophys. Res. Lett., 39, L08105, doi:10.1029/2012GL051393.

Bortnik, J., R. M. Thorne, and N. P. Meredith (2008), The unexpected origin of plasmaspheric hiss from discrete chorus emissions, Nature, 452, 62-66, doi:10.1038/nature06741.

Bortnik, J., L. Chen, W. Li, R. M. Thorne, and R. B. Horne (2011), Modeling the evolution of chorus waves into plasmaspheric hiss, J. Geophys. Res., 116, A08221, doi:10.1029/2011JA016499.

Boskova, J., F. Jiricek, P. Triska, B. V. Lundin, and D. R. Shkliar (1990), A possible common nature of equatorial half-gyrofrequency VLF emissions and discrete plasmaspheric emissions, Ann. Geophys., 8, 755-763.

Bourdarie, S., D. Boscher, T. Beutier, J.-A. Sauvaud, and M. Blanc (1996), Magnetic storm modeling in the Earth's electron belt by the Salammbô code, J. Geophys. Res., 101, 27,171-27,176, doi:10.1029/96JA02284.

Breuillard, H., Y. Zaliznyak, V. Krasnoselskikh, O. Agapitov, A. Artemyev, and G. Rolland (2012), Chorus wave-normal statistics in the Earth's radiation belts from ray tracing technique, Ann. Geophys., in press.

Bunch, N. L., M. Spasojevic, and Y. Y. Shprits (2012), Off-equatorial chorus occurrence and wave amplitude distributions as observed by the Polar Plasma Wave Instrument, J. Geophys. Res., 117, A04205, doi:10.1029/2011JA017228

Chen, Y., G. D. Reeves, and R. H. W. Friedel (2007), The energization of relativistic electrons in the outer Van Allen radiation belt, Nat. Phys., 3, 614-617, doi:10.1038/nphys655.

Cornilleau-Wehrlin, N., et al. (2003), First results obtained by the Cluster STAFF experiment, Ann. Geophys., 21, 437-456, doi:10.5194/angeo21-437-2003.

Cully, C. M., J. W. Bonnell, and R. E. Ergun (2008), THEMIS observations of long-lived regions of large-amplitude whistler waves in the inner magnetosphere, Geophys. Res. Lett., 35, L17S16, doi:10.1029/ 2008GL033643.

Dubyagin, S., V. Sergeev, S. Apatenkov, V. Angelopoulos, A. Runov, R. Nakamura, W. Baumjohann, J. McFadden, and D. Larson (2011), Can flow bursts penetrate into the inner magnetosphere?, Geophys. Res. Lett., 38, L08102, doi:10.1029/2011GL047016.

Dungey, J. W. (1963), Loss of Van Allen electrons due to whistlers, Planet. Space Sci., 11, 591, doi:10.1016/0032-0633(63)90166-1.

Fok, M.-C., R. B. Horne, N. P. Meredith, and S. A. Glauert (2008), Radiation belt environment model: Application to space weather nowcasting, J. Geophys. Res., 113, A03S08, doi:10.1029/2007JA012558.

Friedel, R. H. W., and A. Korth (1996), A dynamic data-driven radiation belt model based on CRRES data, Eur. Space Agency Spec. Publ., ESA-SP 392, 143-150. 
Ginzburg, V. L., and A. A. Rukhadze (1975), Waves in Magnetoactive Plasma, 2nd ed., Nauka, Moscow.

Glauert, S. A., and R. B. Horne (2005), Calculation of pitch angle and energy diffusion coefficients with the PADIE code, J. Geophys. Res., 110, A04206, doi:10.1029/2004JA010851.

Hamlin, D. A., R. Karplus, R. C. Vik, and K. M. Watson (1961), Mirror and azimuthal drift frequencies for geomagnetically trapped particles, J. Geophys. Res., 66, 1-4, doi:10.1029/JZ066i001p00001.

Haque, N., M. Spasojevic, O. Santolík, and U. S. Inan (2010), Wave normal angles of magnetospheric chorus emissions observed on the Polar spacecraft, J. Geophys. Res., 115, A00F07, doi:10.1029/2009JA014717.

Horne, R. B., R. M. Thorne, S. A. Glauert, J. M. Albert, N. P. Meredith, and R. R. Anderson (2005), Timescale for radiation belt electron acceleration by whistler mode chorus waves, J. Geophys. Res., 110, A03225, doi:10.1029/2004JA010811.

Ingraham, J. C., T. E. Cayton, R. D. Belian, R. A. Christensen, R. H. W. Friedel, M. M. Meier, G. D. Reeves, and M. Tuszewski (2001), Substorm injection of relativistic electrons to geosynchronous orbit during the great magnetic storm of March 24, 1991, J. Geophys. Res., 106, 25,759-25,776, doi:10.1029/2000JA000458.

Jirícek, F., D. R. Shklyar, and P. Tríska (2001), LHR effects in nonducted whistler propogation - new observations and numerical modelling, Ann. Geophys., 19, 147-157, doi:10.5194/angeo-19-147-2001.

Kennel, C. F., and H. E. Petschek (1966), Limit on stably trapped particle fluxes, J. Geophys. Res., 71, 1-28.

Li, W., J. Bortnik, R. M. Thorne, and V. Angelopoulos (2011), Global distribution of wave amplitudes and wave normal angles of chorus waves using THEMIS wave observations, J. Geophys. Res., 116, A12205, doi:10.1029/2011JA017035.

Lyons, L. R. (1974), Pitch angle and energy diffusion coefficients from resonant interactions with ion-cyclotron and whistler waves, J. Plasma Phys., 12, 417-432, doi:10.1017/S002237780002537X.

Lyons, L. R., and D. J. Williams (1984), Quantitative Aspects of Magnetospheric Physics, D. Reidel, Dordrecht, Netherlands.

Lyons, L. R., R. M. Thorne, and C. F. Kennel (1972), Pitch-angle diffusion of radiation belt electrons within the plasmasphere, J. Geophys. Res., 77, 3455-3474, doi:10.1029/JA077i019p03455.

Meredith, N. P., R. B. Horne, and R. R. Anderson (2001), Substorm dependence of chorus amplitudes: Implications for the acceleration of electrons to relativistic energies, J. Geophys. Res., 106, 13,165-13,178, doi:10.1029/2000JA900156.

Meredith, N. P., R. B. Horne, R. M. Thorne, and R. R. Anderson (2003) Favored regions for chorus-driven electron acceleration to relativistic energies in the Earth's outer radiation belt, Geophys. Res. Lett., 30(16), 1871, doi:10.1029/2003GL017698.

Meredith, N. P., R. B. Horne, R. M. Thorne, D. Summers, and R. R. Anderson (2004), Substorm dependence of plasmaspheric hiss, J. Geophys. Res., 109, A06209, doi:10.1029/2004JA010387.

Mourenas, D., and J.-F. Ripoll (2012), Analytical estimates of quasi-linear diffusion coefficients and electron lifetimes in the inner radiation belt, J. Geophys. Res., 117, A01204, doi:10.1029/2011JA016985.

Mourenas, D., A. V. Artemyev, J.-F. Ripoll, O. V. Agapitov, and V. V. Krasnoselskikh (2012), Timescales for electron quasi-linear diffusion by parallel and oblique lower-band Chorus waves, J. Geophys. Res., 117, A06234, doi:10.1029/2012JA017717.

Ni, B., R. M. Thorne, N. P. Meredith, Y. Y. Shprits, and R. B. Horne (2011), Diffuse auroral scattering by whistler mode chorus waves: Dependence on wave normal angle distribution, J. Geophys. Res., 116, A10207, doi:10.1029/2011JA016517.
Orlova, K. G., and Y. Y. Shprits (2010), Dependence of pitch-angle scattering rates and loss timescales on the magnetic field model, Geophys. Res. Lett., 37, L05105, doi:10.1029/2009GL041639.

Pokhotelov, D., F. Lefeuvre, R. B. Horne, and N. Cornilleau-Wehrlin (2008), Survey of ELF-VLF plasma waves in outer radiation belt observed by Cluster STAFF-SA experiment, Ann. Geophys., 26, 3269-3277, doi:10.5194/angeo-26-3269-2008.

Reinisch, B. W., X. Huang, P. Song, J. L. Green, S. F. Fung, V. M. Vasyliunas, D. L. Gallagher, and B. R. Sandel (2004), Plasmaspheric mass loss and refilling as a result of a magnetic storm, J. Geophys. Res., 109, A01202, doi:10.1029/2003JA009948.

Santolík, O., D. A. Gurnett, J. S. Pickett, J. Chum, and N. Cornilleau-Wehrlin (2009), Oblique propagation of whistler mode waves in the chorus source region, J. Geophys. Res., 114, A00F03, doi:10.1029/2009JA014586.

Sazhin, S. S., and R. B. Horne (1990), Quasilongitudinal approximation for whistler-mode waves in the magnetospheric plasma, Planet Space Sci. 38, 1551-1553, doi:10.1016/0032-0633(90)90160-R.

Sheeley, B. W., M. B. Moldwin, H. K. Rassoul, and R. R. Anderson (2001), An empirical plasmasphere and trough density model: CRRES observations, J. Geophys. Res., 106, 25,631-25,642, doi:10.1029/2000JA000286.

Shklyar, D., and H. Matsumoto (2009), Oblique whistler-mode waves in the inhomogeneous magnetospheric plasma: Resonant interactions with energetic charged particles, Surv. Geophys., 30, 55-104, doi:10.1007/s10712009-9061-7.

Shklyar, D. R., and F. Jiřriček (2000), Simulation of nonducted whistler spectrograms observed aboard the MAGION 4 and 5 satellites, J. Atmos. Sol. Terr. Phys., 62, 347-370, doi:10.1016/S1364-6826(99)00097-8.

Shprits, Y. Y., and B. Ni (2009), Dependence of the quasi-linear scattering rates on the wave normal distribution of chorus waves, J. Geophys. Res., 114, A11205, doi:10.1029/2009JA014223.

Shprits, Y. Y., R. M. Thorne, R. B. Horne, and D. Summers (2006), Bounce-averaged diffusion coefficients for field-aligned chorus waves, J. Geophys. Res., 111, A10225, doi:10.1029/2006JA011725.

Shprits, Y. Y., N. P. Meredith, and R. M. Thorne (2007), Parameterization of radiation belt electron loss timescales due to interactions with chorus waves, Geophys. Res. Lett., 34, L11110, doi:10.1029/2006GL029050.

Shprits, Y. Y., D. A. Subbotin, N. P. Meredith, and S. R. Elkington (2008), Review of modeling of losses and sources of relativistic electrons in the outer radiation belt II: Local acceleration and loss, J. Atmos. Sol. Terr. Phys., 70, 1694-1713, doi:10.1016/j.jastp.2008.06.014.

Su, Z., H. Zheng, and S. Wang (2010), Three-dimensional simulation of energetic outer zone electron dynamics due to wave-particle interaction and azimuthal advection, J. Geophys. Res., 115, A06203, doi:10.1029/ 2009JA014980.

Subbotin, D., Y. Shprits, and B. Ni (2010), Three-dimensional VERB radiation belt simulations including mixed diffusion, J. Geophys. Res., 115, A03205, doi:10.1029/2009JA015070.

Summers, D. (2005), Quasi-linear diffusion coefficients for field-aligned electromagnetic waves with applications to the magnetosphere, J. Geophys. Res., 110, A08213, doi:10.1029/2005JA011159.

Summers, D., B. Ni, and N. P. Meredith (2007), Timescales for radiation belt electron acceleration and loss due to resonant wave-particle interactions: 1. Theory, J. Geophys. Res., 112, A04206, doi:10.1029/2006JA011801.

Trakhtengerts, V. Y. (1966), Stationary states of the Earth's outer radiation zone, Geomagn. Aeron., 6, 827-836.

Varotsou, A., D. Boscher, S. Bourdarie, R. B. Horne, N. P. Meredith, S. A. Glauert, and R. H. Friedel (2008), Three-dimensional test simulations of the outer radiation belt electron dynamics including electronchorus resonant interactions, J. Geophys. Res., 113, A12212, doi:10.1029/2007JA012862. 